\title{
19. CENOZOIC RADIOLARIA FROM LEG 1
}

\author{
W. R. Riedel, Scripps Institution of Oceanography, La Jolla, California \\ and \\ J. D. Hays, Lamont-Doherty Geological, Observatory, Palisades, New York
}

Cenozoic radiolarian samples were taken at one level each at drilling Sites 3 and 7, and nine levels at Site 6.

At Site 3 (Sample 1-3-2-3-133-134) the radiolarians are very rare, and evidently reworked. There are well preserved Eocene forms including Podocyrtis papalis, Dictyophimus babylonis, and questionable Phormocyrtis embolum, and also some poorly preserved Dictyomitra-like stichocyrtids which appear to be Cretaceous.

At Site 6, radiolarians occur in an Eocene sequence characterized by numerous graded beds, apparently turbidites. The turbidity currents evidently did not bring to this locality any radiolarians older than Eocene, but they probably caused mixing of microfossils from different parts of the Eocene. The radiolarians are well preserved. In most samples they are accompanied by diatoms, and in some samples they are almost masked by sponge spicules (Plate 1, A \& B).

The occurrences of some of the more easily recognizable radiolarians in the samples examined are shown in Table 1. Artophormis barbadiensis (Ehrenberg) and Calocyclas turris Ehrenberg, both of which have been recorded only in late Eocene assemblages, were not found in any of the samples. Because of the great amount of reworking in this sequence, it is difficult to determine which forms are of the same age as the sediment, and which are older forms transported into this locality. Many of the species identified occur throughout the section, but some are restricted to the upper samples and their lower limits may prove useful in correlating with Eocene sequences elsewhere. Lithocyclia ocellus is one such species: it is not yet possible to postulate a form ancestral to it.

Podocyrtis papalis (Plate 1, C) does not occur in the lowest samples examined, and it seems to be preceded (and for a time accompanied) by a form here designated as Podocyrtis sp. $a$. This form differs from $P$. papalis in having an externally expressed lumbar stricture, and toothed feet (Plate 1, D) which are not well separated in some specimens. Podocyrtis sp. $a$ is not to be confused with a form (Plate 1, E) occurring throughout this sequence which may be identified as Podocyrtis sinuosa Ehrenberg, and is distinguished by the abdomen, with a wall structure different from that of the thorax, being generally much longer than the thorax.

Eusyringium fistuligerum occurs in only the uppermost samples and seems to be preceded and accompanied by a two-segmented form (Plate 1, F \& G) which lacks a distal tubular prolongation and which may be Sethocapsa lagena (Ehrenberg).

At Site 7, Eocene radiolarians are evidently in situ. The sample examined is designated as " $1-7-2$ core fragment", and contains numerous, rather corroded skeletons including few Sethamphora mongolfieri, doubtful fragments of Podocyrtis sp. a, rare Anthocyrtium hispidum, few Dictyophimus babylonis, few Sethocapsa lagena, questionable Phormocyrtis embolum few Lithochytris $\mathrm{sp}(\mathrm{p})$., rare Phormocyrtis striata and few doubtful fragments of Podocyrtis sinuosa.

\section{PLATE 1}

A Radiolarians and diatoms. Fraction coarser than $62 \mu$, of the sample at 1-6-3-3-100-102. $\times 70$.

B Sponge spicules, diatoms and radiolarians. Fraction coarser than $62 \mu$, of the sample at 1-6-5-2-78-80. $\times 70$.

C Podocyrtis papalis Ehrenberg, from 1-6-3-4-68-71. X 175.

D Podocyrtis sp. a, 1-6-5-1-5-7.X 175.

E Podocyrtis sinuosa Ehrenberg, from 1-6-3-4-68-71. X 175.

F Sethocapsa lagena (Ehrenberg), from 1-6-3-4-68-71. X 355.

G Sethocapsa lagena (Ehrenberg), from 1-6-5-1-5-7. $\times 355$. 


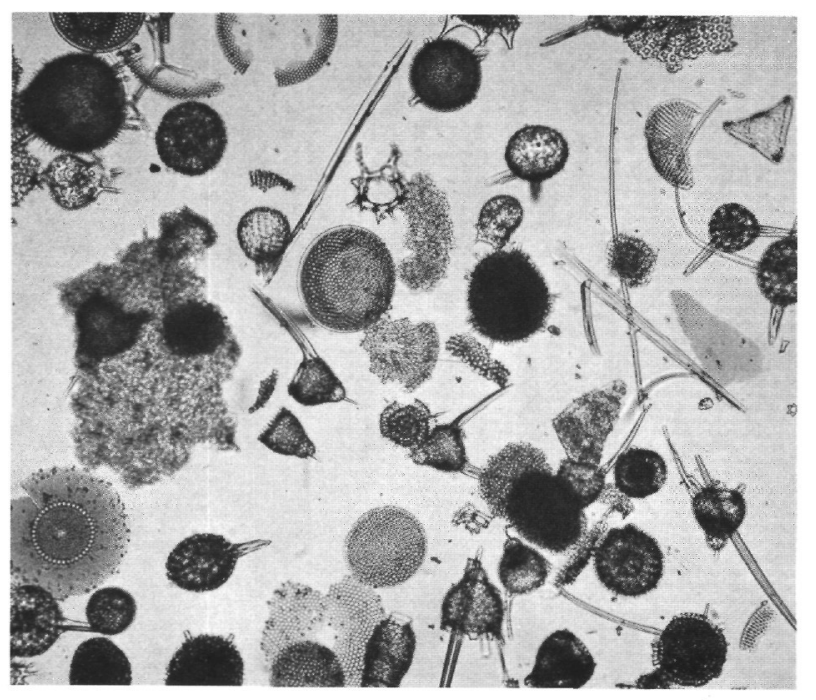

A

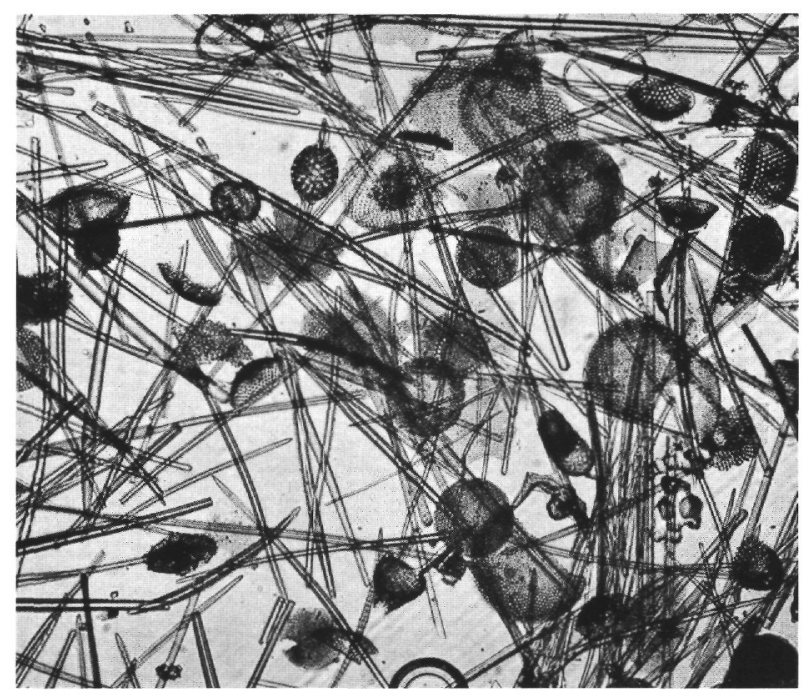

B

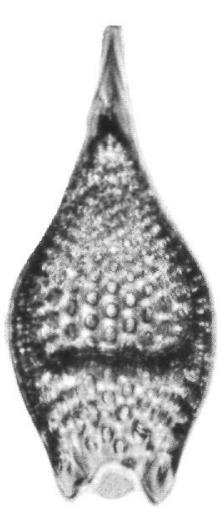

C

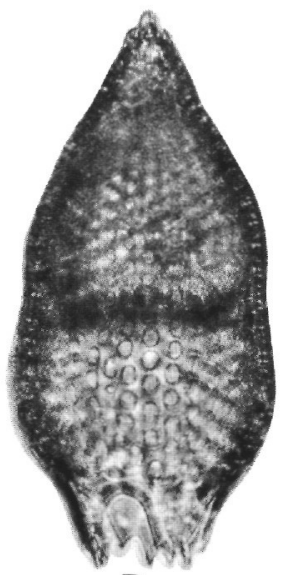

D

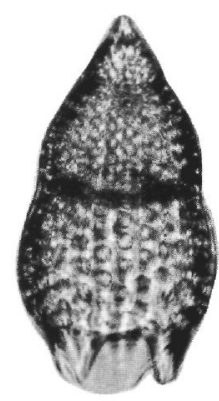

$E$

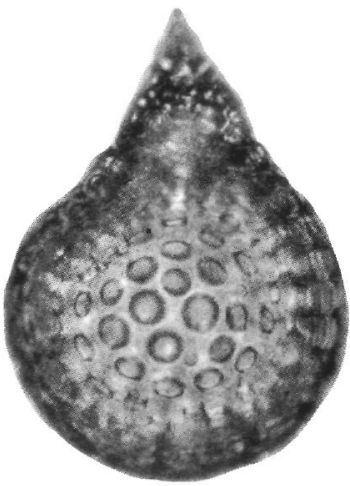

$\mathbf{F}$

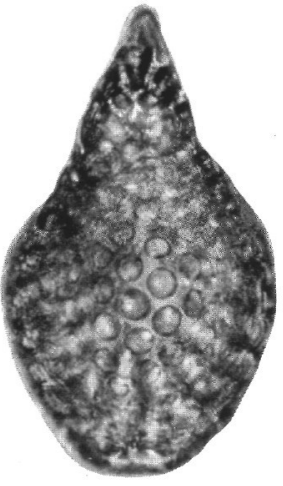

G

Plate 1. Riedel and Hays - Cenozoic Radiolaria - Leg 1. 
TABLE 1

Occurrences of Some Radiolarians at Site $6^{\mathrm{a}}$

\begin{tabular}{|c|c|c|c|c|c|c|c|c|c|c|c|c|c|c|}
\hline & 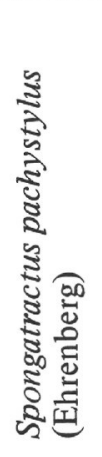 & 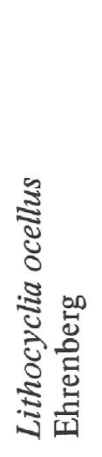 & 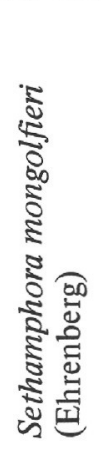 & 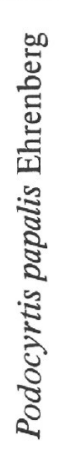 & 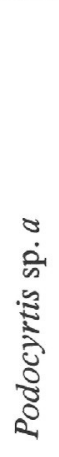 & 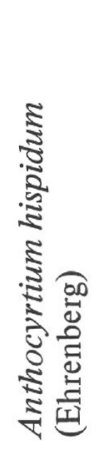 & 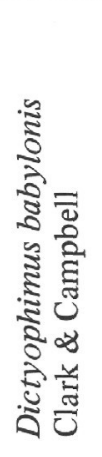 & 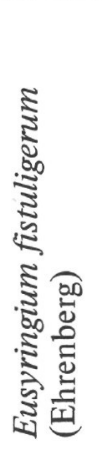 & 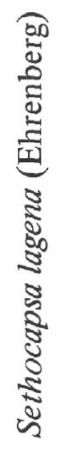 & 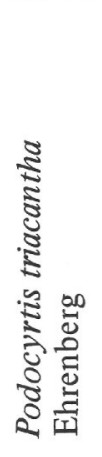 & 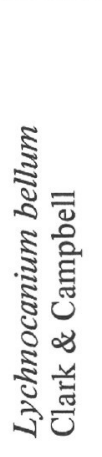 & 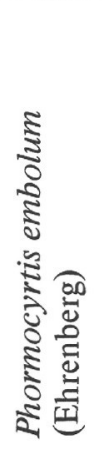 & 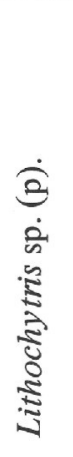 & 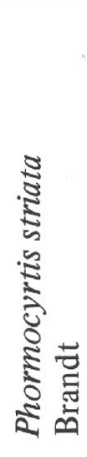 \\
\hline $1-6-3-3-100-102$ & - & $\mathrm{R}$ & $\mathrm{R}$ & $\mathrm{R}$ & - & $\mathrm{C}$ & $\mathrm{F}$ & $\mathrm{F}$ & $\mathrm{R}$ & $\mathrm{R}$ & $\mathrm{R}$ & $\mathrm{C}$ & - & $\mathrm{F}$ \\
\hline $1-6-3-4-68-71$ & $\mathrm{R}$ & $\mathrm{R}$ & $\mathrm{C}$ & $\mathrm{F}$ & - & $\mathrm{F}$ & $\mathrm{F}$ & $\mathrm{R}$ & $\mathrm{F}$ & $\mathrm{F}$ & $\mathrm{F}$ & $\mathrm{C}$ & $\mathrm{F}$ & $\mathrm{F}$ \\
\hline $1-6-4-1-100-101$ & - & $\mathrm{R}$ & $\mathrm{C}$ & - & - & $\mathrm{F}$ & F & - & $\mathrm{R}$ & $\mathrm{R}$ & $\mathrm{R}$ & F & - & $\mathrm{F}$ \\
\hline $1-6-4-2-54-56$ & $\mathrm{R}$ & F & $\mathrm{F}$ & $\mathrm{R}$ & - & $\mathrm{F}$ & $\mathrm{R}$ & - & $\mathrm{F}$ & $\mathrm{R}$ & $\mathrm{F}$ & $\mathrm{F}$ & $\mathrm{R}$ & $\mathrm{F}$ \\
\hline $1-6-4-3-108-111$ & - & $\mathrm{R}$ & $\mathrm{F}$ & - & $\mathrm{F}$ & $\mathrm{F}$ & $\mathrm{F}$ & - & $\mathrm{C}$ & $\mathrm{R}$ & $\mathrm{F}$ & $\mathrm{F}$ & $\mathrm{F}$ & $\mathrm{F}$ \\
\hline $1-6-5-1-5-7$ & - & $\mathrm{R}$ & $\mathrm{C}$ & $\mathrm{R}$ & $\mathrm{R}$ & $\mathrm{F}$ & $\mathrm{C}$ & - & $\mathrm{F}$ & - & $\mathrm{F}$ & $\mathrm{R}$ & $\mathrm{C}$ & $\mathrm{F}$ \\
\hline $1-6-5-2-78-80$ & - & - & $\mathrm{C}$ & $\mathrm{R}$ & - & $\mathrm{R}$ & $\mathrm{F}$ & - & $\mathrm{F}$ & - & $\mathrm{R}$ & $\mathrm{C}$ & - & $\mathrm{R}$ \\
\hline $1-6-6-1-149-151$ & - & - & F & - & F & $\mathrm{C}$ & $\mathrm{F}$ & - & $\mathrm{C}$ & $\mathrm{R}$ & $\mathrm{F}$ & $\mathrm{F}$ & $\mathrm{R}$ & $\mathrm{F}$ \\
\hline $1-6-6-3-50-52$ & - & - & + & - & + & + & + & - & + & . & + & + & - & + \\
\hline
\end{tabular}

${ }_{\mathrm{R}} \mathrm{R}=$ rare, $\mathrm{F}=$ few, $\mathrm{C}=$ common. In the lowest sample examined, radiolarians are few and rather corroded, and therefore only presences and absences are indicated. The sample listed as 1-6-4-1-100-101 is evidently from a different level, since no sample was taken at $100-101 \mathrm{~cm}$ in that section of the core. 